identities was highlighted. A key recommendation from the youth delegation is that organisations continue to not only invite youth participation at technical conferences, but also value their input as near-to-peer educators of young people.

Outcome The youth delegation formed an international alliance for sex education, and recommendations from the technical conference have been distributed to delegates' organisations to inform their future work providing education and sexual healthcare to young people. As an organisation working with an ever more diverse school age population Sexpression will endeavour to make all its teaching resources inclusive of all cultures, sexualities and identities.

\section{G17(P) TRANSITION TO ADULT CARE AUDIT: SUCCESSES AND CHALLENGES AT A TERTIARY CHILDREN'S HOSPITAL}

${ }^{1}$ A Low, ${ }^{1 J}$ Culshaw, ${ }^{2}$ P Narula. 'Department of Paediatrics, Sheffield Children's Hospital, Sheffield, UK; ${ }^{2}$ Department of Paediatric Gastroenterology, Sheffield Children's Hospital, Sheffield, UK

\subsection{6/archdischild-2018-rcpch.16}

Background The period of transition from paediatric to adult care is a risky one. Recent NICE guidelines and the national Ready, Steady, Go (RSG) initiative aim to improve the transition process for young people (YP). We present an overview and audit of current transition services at a tertiary paediatric hospital where a 2016 Care Quality Commission inspection labelled Transition Services as 'Requires improvement.' In response, the hospital has appointed two transition nurses, encouraged the implementation of RSG and set up a transition website for YP to access.

Methods We reviewed two weeks of inpatient data to find patients with medical conditions requiring transition, and compared their care to the NICE recommendations:

- Did discussions around transition begin by age 14 (or at diagnosis)?

- Was there an annual meeting where transition was discussed?

- Was there evidence of a named transition worker?

- Did they meet a practitioner from adult services before transferring?

Results 43 patients were included in our audit, with the oldest being 20 years old. $58 \%$ had started transition, but only $36 \%$ by the age of $14.30 \%$ had an annual review, and $20 \%$ had a named worker. $48 \%$ had met someone from adult services before transition. Of patients with complex problems under three or more specialties, $66 \%$ had transition started but none by the age of 14 . Medical specialties fared better than surgical specialties across all domains.

Conclusion This is the first time this audit has been conducted at this hospital. Our audit demonstrates that despite the recent improvements in transition services, our hospital still faces challenges in meeting the NICE transition guidelines. The patients least likely to meet the guidelines were surgical patients and complex patients under the care of multiple specialties. Further plans include mandatory training on transition, and consideration of $\mathrm{YP}$ using a tablet to access RSG in the waiting room before appointments.

\section{G18(P) OUR CHRONIC FATIGUE SYNDROME/ ENCEPHALOMYELITIS SERVICE IS NICE, BUT IS IT PATIENT FRIENDLY? EXPERIENCE OF GOING BEYOND AUDIT}

AM Taylor, J Man, EJ Parish, T Segal. Children and Young People's Services, University College London Hospitals NHS Foundation Trust, London, UK

10.1136/archdischild-2018-rcpch.17

Aim To assess the quality of diagnosis and management of Chronic Fatigue Syndrome (CFS)/Encephalomyelitis (ME) in a tertiary Service against NICE guidelines, along with views of the young people assessed.

Methods A retrospective audit of patients referred to the CFS/ ME service over a 12 month period (December 2014-December 2015 chosen to allow time for follow up). Case notes and electronic records were reviewed for baseline demographics, NICE criteria (Guideline CG53) and additional information. Patients and/or their parents were contacted for feedback by telephone using a structured transcript and standardised Friends and Family questionnaire.

Results 30 patients were referred to the CFS/ME service, 3 of whom did not attend and were excluded from analysis.

We assessed 5 NICE guideline criteria that relate to young people, all of which have a 100\% standard. Three criteria focus on providing patients with relevant information. $86 \%$ of our patient records documented advice on symptom management, $71 \%$ on work/education, and $43 \%$ on general principles of CFS/ME. The service was $100 \%$ compliant with criterion 4 (a diagnosis of CFS/ME should only be made when symptoms have persisted for 3 months). The final criterion assesses how many patients with mild/moderate $\mathrm{CFS} / \mathrm{ME}$ are provided with cognitive behavioural therapy and/or graded exercise therapy; $73 \%$ of patients received one of these.

13 of the 27 young people/parents contacted for the Friends and Family questionnaire answered the telephone, 3 did not answer the questions. Of the 10 respondents $(8$ parents and 2 young people), 5 'agreed' with the statement 'this is a good service for my friends and family to be looked after in if they needed similar treatment or care', 3 'agreed a bit' and 2 'disagreed a lot'. Improvements focused on practicalities such as distance to the hospital, paucity of specialist services, and insufficient access to therapies.

Conclusion Our Service appropriately diagnoses young people with CFS/ME though compliance with NICE guidelines could be improved. We were disappointed that feedback was predominantly parental and wonder if an online or written Friends and Family test would facilitate hearing from young people themselves.

\section{G19(P) WORKING IN PARTNERSHIP WITH ADOLESCENT SMOKERS TO IMPROVE THE COMMUNICATION SKILLS OF MEDICAL STUDENTS}

1I Peppas, ${ }^{2}$ EJA Fitchett, 'W Kenworthy, ${ }^{1} \mathrm{P}$ Kimkool, ${ }^{1} \mathrm{CR}$ Fertleman. 'Department of Paediatrics, Whittington Health, London, UK; ${ }^{2}$ University College London Great Ormond Street Institute of Child Health, London, UK

10.1136/archdischild-2018-rcpch.18 